\title{
Characterization of Pu-bearing materials by non-destructive spectrometric approach: relevance for nuclear forensics
}

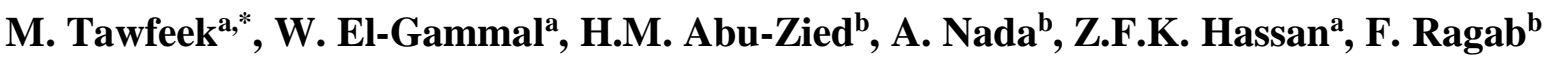 \\ ${ }^{a}$ Nuclear and Radiological Regulatory Authority (ENRRA), Cairo, Egypt. \\ ${ }^{b}$ Faculty of Women for Arts, Science and Education, Ain Shams university, Cairo, Egypt.
}

\begin{abstract}
One of the main components that should be in place to control nuclear materials and their applications is a robust measurement system. The function of this system is not only to verify the correctness of the information regarding nuclear materials but it must be capable to characterize the unknown materials as well. The material's chemical and physical attributes concluded from the characterization process are needed to determine the intended use and origin of such materials. Thus, the characterization of nuclear materials is a key issue and equally important in nuclear safeguards (for inventory verification) and in nuclear security (for forensics attribution). Herein, an absolute spectrometric non-destructive analysis (NDA) method was used to characterize some plutonium-bearing materials. The gamma-ray spectra from fifteen samples were obtained using a high purity germanium detector. A portable HPGebased radionuclide (Falcon 5000) with the In-Situ Object Counting System (ISOCS) which is based on a pre-efficiency calibrated high-resolution gamma-spectrometer in combination with analysis software was applied. The isotopic composition of the assayed samples was estimated from the direct measurement of the activities of $\mathrm{Pu}$ isotopes as well as $\mathrm{Am}, \mathrm{Pa}$, and $\mathrm{U}$ isotopes. The obtained results have shown that the proposed spectrometric method can be successfully used to characterize $\mathrm{Pu}$-bearing samples for nuclear forensic purposes.
\end{abstract}

Key words: Plutonium, Non-destructive analysis, Nuclear forensics, Gamma spectrometry.

\section{Introduction}

Nuclear forensics (NF) is usually used to describe the technical means used to characterize various nuclear and other radioactive materials and interpret the resulting data. This science is critical in responding to trafficking activities, deterring nuclear security-related events, and verifying that international treaties (e.g. Non-Proliferation Treaty) are being upheld. NF aims at re-establishing the history and origin of the seized material, using a broad array of advanced physical, chemical, and isotopic procedures to characterize sampled or seized nuclear or other radioactive materials. It is based on indicators that arise from known relationships between material characteristics and process history. NF analysis includes the categorization and

*Corresponding author: M. Tawfeek, Nuclear and Radiological Regulatory Authority (ENRRA), Cairo, Egypt.

E-mail: firestorm18@yahoo.com 
characterization of the seized material and correlation with production history followed by interpretation [1].

Plutonium $(\mathrm{Pu})$ is formed as a by-product in nuclear reactors through neutron capture of uranium and subsequent $\beta$-decays, as the following:

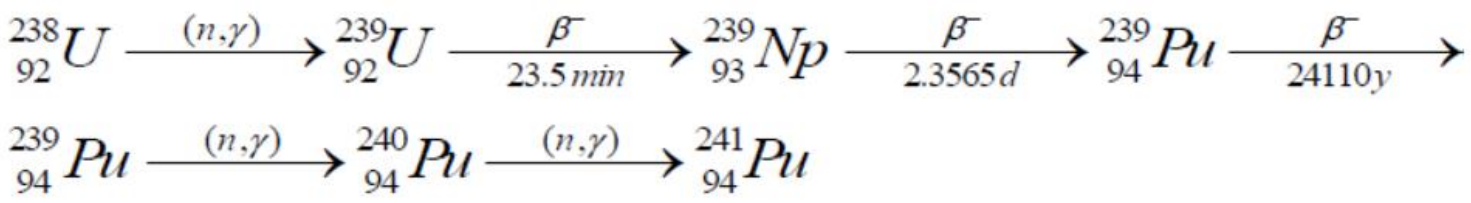

Reactor-produced $\mathrm{Pu}$ contains information from which the history of the material can be deduced. This includes the age, the production mode (reactor type), the processing method (as deduced from trace impurities), and the intended use (from the isotopic composition). The primary focus of NF investigations of seized Pu material is to specify correctly its origin. This could be achieved via determination of the reactor type where $\mathrm{Pu}$ was produced, which may allow one to trace back its origin. To this end, an isotope correlation has been established, which depends upon the reactor type. Therefore, several analytical techniques had been developed to measures accurately the built-up daughter nuclides and determine the age of the $\mathrm{Pu}$ material. A methodology has been developed to determine Pu age in samples by thermal ionization mass spectrometry using independent parent/daughter relations. This has been demonstrated for reference materials of known ages as well as for real samples and compared to gamma spectrometry [2]. Some scientists developed a radiochemical procedure for the determination of ${ }^{241} \mathrm{Pu}$ in environmental samples, based on the use of extraction chromatography resins. Their measurements showed clear evidence that the Pu present in the soil originated from global fallout from nuclear weapons tests [3]. Others presented a fingerprinting procedure, based on the direct use of $\mathrm{Pu}$ isotopic ratios to discriminate spent fuels of different origin [4].

Gamma spectrometry is used in NF analysis as it offers a non-destructive capability thereby preserving the recovered sample for another testing. It is applied as the initial measurement technique on receipt of the unknown, recovered nuclear materials enabling rapid characterization of the content and, if applicable, isotopic composition. Usually, upon discovery or seizure of illicitly trafficked materials, the unknown specimen should be measured via gamma spectrometry within 24-hours to gain a rapid but preliminary understanding of the radiological composition and concentration.

Determining the composition of a sample's key components (e.g. U, $\mathrm{Pu}$, and progeny isotopes) is clearly of central importance. The age of seized nuclear material is a very important 
parameter for the origin determination because it may serve to exclude certain production or reprocessing plants. Additionally, dating techniques can be used to identify procedures that may have been carried out on that material, e.g. reprocessing. The age can be determined by several techniques, among them, gamma-spectrometry of the ${ }^{241} \mathrm{Pu} /{ }^{241} \mathrm{Am}$ ratio appears as a fast method which does not require chemical separation [5]

This study involves the characterization and isotopic composition analysis based on the direct activity measurement of $\mathrm{Pu}$ samples using the non-destructive gamma spectrometric method.

\section{Experimental setup:}

The ISOCS technique was introduced into the Agency's activities just a few years ago, and this methodology is being expanded to more and more NDA measurement techniques.

There is no NDA technique which is capable to verify as wide range of NM types with different matrices, chemical and physical forms, isotopic compositions and container shapes as ISOCS technique. Fresh and slightly irradiated fuel elements, inventory items, clean and dirty scraps, wastes and hold-up materials. ISOCS calculations were performed to estimate the absolute efficiency of the detector at the sample location with respect to the detector.

In this work, an ISOCS system has been used to measure the Pu samples. Falcon 5000 unit was used with model S573 ISOCS calibration software. The unit incorporates a HPGe detector with $60 \times 30 \mathrm{~mm}$ dimensions for spectrometry with energy range between $20 \mathrm{keV}$ to 3.0MeV.

Fifteen samples of thin layer of Pu precipitated on discs with diameter of $3.4 \mathrm{~cm}$ and active diameter of $1 \mathrm{~cm}$ were measured. The sample preparation method is unknown, since these samples are orphan. There is no difference between the samples, they all have the same physical and chemical characteristics, except the elements percentage. Each sample was measured in the way that placed directly on the center of the detector crystal. Since the measured material is a very thin layer, self-attenuation due to material thickness and matrix material was neglected. The average measuring life times was 22 hours and the dead time was always less the $1 \%$.

Specific gamma ray energies, which are a signature for interested isotopes have been measured in understudy samples as illustrate in figure $\mathbf{1}$ and gamma ray energies for interested isotopes in understudy samples have been illustrated in table $\mathbf{1}$. 
Table (1): Gamma ray energy lines for the interested isotopes in understudy samples

\begin{tabular}{|c|c|c|}
\hline Isotope & Gamma energy line & I (\%) \\
\hline $\mathrm{Pu}^{239}$ & 129.29 & 0.0063 \\
\hline $\mathrm{Am}^{241}$ & 59.54 & 35.9 \\
\hline $\mathrm{Pu}^{241}$ & 148.57 & 0.00019 \\
\hline $\mathrm{Pu}^{242}$ & 158.8 & 0.00045 \\
\hline $\mathrm{U}^{235}$ & 185.7 & 57.2 \\
\hline $\mathrm{Pa}^{234 \mathrm{~m}}$ & 1001 & 0.59 \\
\hline
\end{tabular}

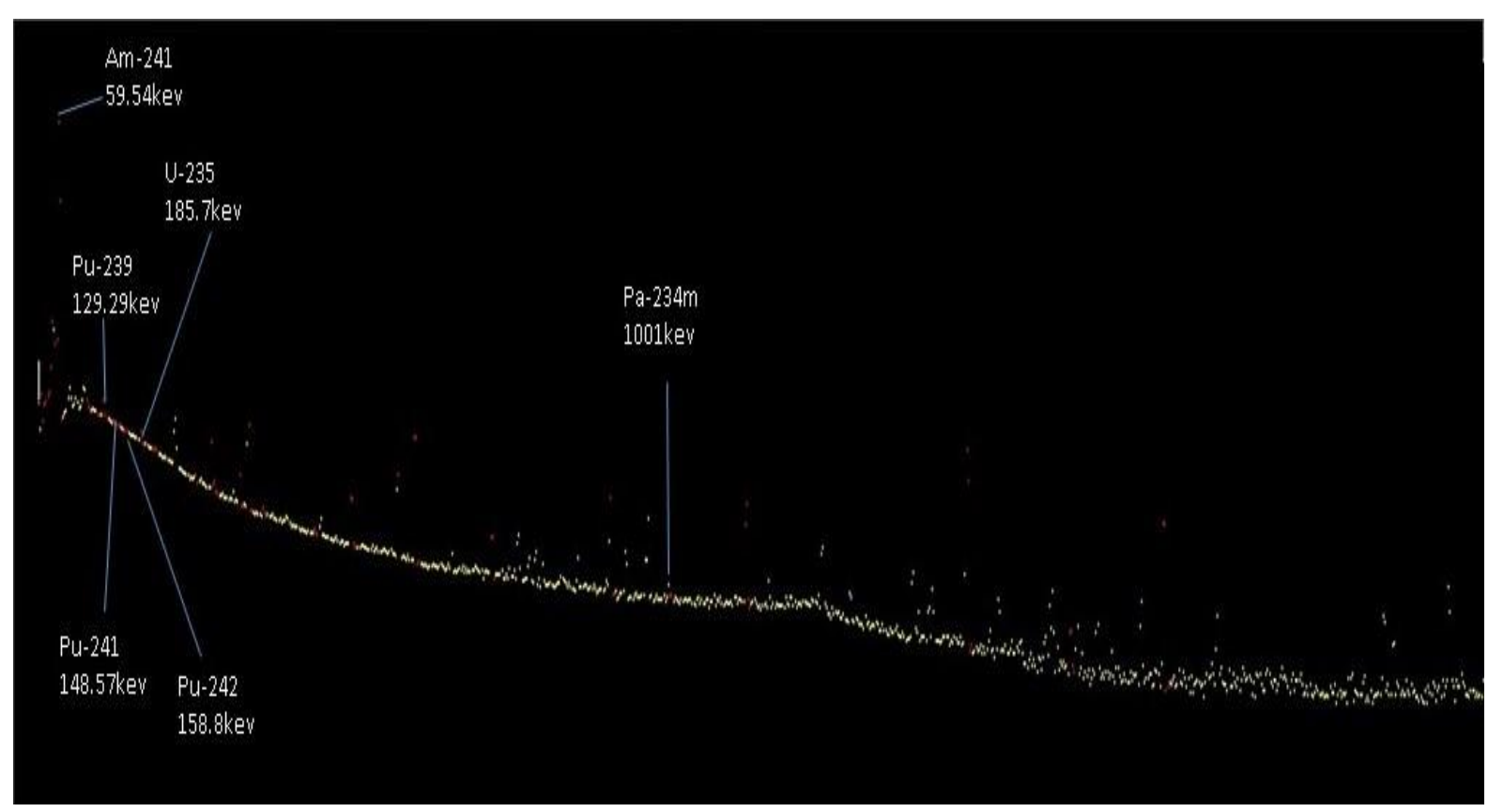

Figure 1: Specific gamma ray energies lines of the interested isotopes in the samples understudy.

All measurements were carried out by adjusting the experimental setup in such a way that errors due to electronic losses were minimized (dead time did not exceed $2 \%$ ). Also, the measuring life time was optimized to achieve good statistics (statistical errors are always kept below $1 \%)$. 


\section{Results and discussion}

The ISOCS detector characterization method uses mathematical techniques to determine gamma-ray full energy peak efficiencies of germanium detectors at an arbitrary energy and spatial location within the characterization range.

The first and the major information we can get directly from the NDA is the activity concentrations of the different isotopes exist in the samples. The calculated activity for the radionuclide measured using a gamma radiation detector is given by:

$$
\boldsymbol{A}=\frac{C}{\varepsilon \cdot I}=B \boldsymbol{q}
$$

where:

$\boldsymbol{C}$ is the count rate of a specific gamma line energy;

$\boldsymbol{\varepsilon}$ is absolute efficiency;

and $\boldsymbol{I}$ is the branching ratio of the measured energy.

with total error given by:

$$
\sigma=\sqrt{\frac{\sigma_{x}^{2}+\sigma_{y}^{2}}{2}}
$$

where:

$\boldsymbol{\sigma}_{\boldsymbol{x}}$ is the error in area under the curve of spicific gamma line energy peak;

and $\sigma_{\boldsymbol{y}}$ is the statistical error of the same gamma line peak.

The measured activity concentrations in $(\mathrm{Bq})$ of different isotopes in the samples under investigation are listed in table 2 . The average activity concentrations ratios of the measured isotopes in the samples are shown in figure2. 
Table 2: The activity concentrations (Activity \pm error \%) of the measured radioisotopes in $(B q)$.

\begin{tabular}{|c|c|c|c|c|c|c|}
\hline $\begin{array}{c}\text { Sample } \\
\text { codes }\end{array}$ & ${ }^{*} \mathbf{P u}^{241}$ & ${ }^{*} \mathrm{Am}^{241}$ & $\mathbf{P u}^{239}$ & $\mathbf{P u}^{242}$ & $\mathbf{U}^{235}$ & $\mathbf{P a}^{234 m}$ \\
\hline N6140 & $2312.700 \pm 2.039$ & $498.311 \pm 1.810 \mathrm{E}-3$ & $3868.543 \pm 19.180 \mathrm{E}-3$ & $6973.248 \pm 0.125$ & $0.518 \pm 1.737$ & $\begin{array}{c}31.778 \pm 6 \\
290 \mathrm{E}-3\end{array}$ \\
\hline N6141 & $1212.170 \pm 7.889$ & $462.523 \pm 1.950 \mathrm{E}-3$ & $3552.480 \pm 21.450 \mathrm{E}-3$ & $2617.502 \pm 0.314$ & $0.591 \pm 1.354$ & $\begin{array}{l}16.909 \pm 1 \\
1.830 \mathrm{E}-3\end{array}$ \\
\hline N6142 & $1799.019 \pm 9.604$ & $487.581 \pm 1.850 \mathrm{E}-3$ & $3891.551 \pm 18.580 \mathrm{E}-3$ & $9196.286 \pm 0.65$ & $0.636 \pm 1.415$ & $\begin{array}{c}29.193 \pm 6 \\
850 \mathrm{E}-3\end{array}$ \\
\hline N6144 & $913.794 \pm 37.181$ & $455.971 \pm 1.970 \mathrm{E}-3$ & $3653.836 \pm 20.360 \mathrm{E}-3$ & $5960.040 \pm 0.138$ & $0.581 \pm 1.549$ & $\begin{array}{c}22.357 \pm 8 \\
950 \mathrm{E}-3\end{array}$ \\
\hline N6146 & $3166.290 \pm 3.050$ & $699.808 \pm 1.290 \mathrm{E}-3$ & $4687.107 \pm 16.170 \mathrm{E}-3$ & $11704.600 \pm 0.065$ & $0.568 \pm 1.585$ & $\begin{array}{l}13.693 \pm 1 \\
4.610 \mathrm{E}-3\end{array}$ \\
\hline N6147 & $1388.307 \pm 17.866$ & $721.063 \pm 1.250 \mathrm{E}-3$ & $5301.517 \pm 13.710 \mathrm{E}-3$ & $7066.508 \pm 0.085$ & $0.537 \pm 1.676$ & $\begin{array}{c}22.076 \pm 9 \\
060 \mathrm{E}-3\end{array}$ \\
\hline N6148 & $1641.641 \pm 3.945$ & $663.191 \pm 1.360 \mathrm{E}-3$ & $5481.910 \pm 13.410 \mathrm{E}-3$ & $4542.551 \pm 0.195$ & $0.601 \pm 1.498$ & $\begin{array}{c}28.181 \pm 7 \\
100 \mathrm{E}-3\end{array}$ \\
\hline N6149 & $1710.803 \pm 7.269$ & $761.991 \pm 1.180 \mathrm{E}-3$ & $6328.938 \pm 11.340 \mathrm{E}-3$ & $6955.403 \pm 0.079$ & $0.601 \pm 1.498$ & $\begin{array}{c}31.957 \pm 6 \\
260 \mathrm{E}-3\end{array}$ \\
\hline N6150 & $2861.305 \pm 3.995$ & $653.273 \pm 1.380 \mathrm{E}-3$ & $4800.589 \pm 15.100 \mathrm{E}-3$ & $6583.308 \pm 0.075$ & $0.587 \pm 1.533$ & $\begin{array}{c}36.623 \pm 5 \\
460 \mathrm{E}-3\end{array}$ \\
\hline N6151 & $1186.721 \pm 10.635$ & $622.142 \pm 1.450 \mathrm{E}-3$ & $5225.809 \pm 14.720 \mathrm{E}-3$ & $3975.193 \pm 0.214$ & $0.597 \pm 1.508$ & $\begin{array}{c}26.023 \pm 7 \\
690 \mathrm{E}-3\end{array}$ \\
\hline N6152 & $1461.998 \pm 12.405$ & $715.557 \pm 1.260 \mathrm{E}-3$ & $5190.184 \pm 14.200 \mathrm{E}-3$ & $6668.136 \pm 0.084$ & $0.658 \pm 1.368$ & $\begin{array}{c}35.713 \pm 5 \\
600 \mathrm{E}-3\end{array}$ \\
\hline N6153 & $4141.182 \pm 2.513$ & $1479.785 \pm 6.082 \mathrm{E}-4$ & $10243.510 \pm 7.330 \mathrm{E}-3$ & $4925.228 \pm 0.152$ & $0.579 \pm 1.554$ & $\begin{array}{c}35.505 \pm 5 \\
630 \mathrm{E}-3\end{array}$ \\
\hline
\end{tabular}




\begin{tabular}{|c|c|c|c|c|c|c|}
\hline N6154 & $2555.212 \pm 4.286$ & $1296.603 \pm 6.941 \mathrm{E}-4$ & $8537.243 \pm 8.760 \mathrm{E}-3$ & $6619.167 \pm 0.089$ & $0.553 \pm 1.627$ & $\begin{array}{c}21.224 \pm 9 . \\
42 \mathrm{E}-3\end{array}$ \\
\hline N6155 & $3404.874 \pm 1.866$ & $1266.266 \pm 7.108 \mathrm{E}-4$ & $8036.945 \pm 9.200 \mathrm{E}-3$ & $3250.399 \pm 0.246$ & $0.688 \pm 1.308$ & $\begin{array}{c}25.349 \pm 7 \\
890 \mathrm{E}-3\end{array}$ \\
\hline N6156 & $5922.461 \pm 0.989$ & $1312.565 \pm 6.857 \mathrm{E}-4$ & $8980.881 \pm 8.330 \mathrm{E}-3$ & $11431.200 \pm 0.042$ & $0.623 \pm 1.445$ & $\begin{array}{c}40.272 \pm 4 \\
970 \mathrm{E}-3\end{array}$ \\
\hline Range & $\begin{array}{c}913.794 \pm 37.181 \\
- \\
\mathbf{5 9 2 2 . 4 6 1} \pm \mathbf{0 . 9 8 9}\end{array}$ & $\begin{array}{c}455.971 \pm 1.970 \mathrm{E}-3 \\
- \\
1479.785 \pm 6.082 \mathrm{E}-4\end{array}$ & $\begin{array}{c}3552.480 \pm 21.450 \mathrm{E}-3 \\
- \\
10243.510 \pm 7.330 \mathrm{E}-3\end{array}$ & $\begin{array}{c}2617.502 \pm 0.314 \\
- \\
11704.600 \pm 0.065\end{array}$ & $\begin{array}{c}0.518 \pm 1.737 \\
- \\
0.688 \pm 1.308\end{array}$ & $\begin{array}{c}13.693 \pm 1 \\
4.610 \mathrm{E}-3 \\
- \\
40.272 \pm 4 . \\
970 \mathrm{E}-3\end{array}$ \\
\hline Average & $2378.565 \pm 8.369$ & $\begin{array}{c}\text { 806.442 } \pm 1.295 \mathrm{E}- \\
3\end{array}$ & $\begin{array}{c}5852.069 \pm 14.123 E \\
-3\end{array}$ & $6564.585 \pm 0.131$ & $0.594 \pm 1.510$ & $\begin{array}{c}27.790 \pm \\
7.840 \mathrm{E}- \\
3\end{array}$ \\
\hline
\end{tabular}

$*[5]$.

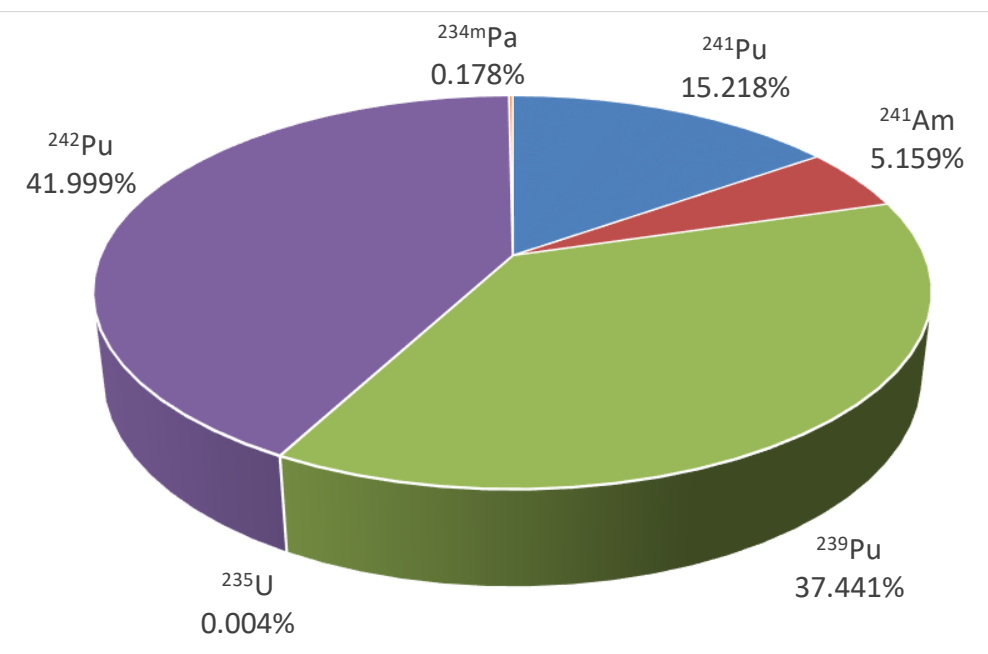

Figure 2: The average activity concentrations ratios of the measured isotopes in the samples.

From figure 2, it is clear that most of the activity in the samples are coming from $\mathbf{P u}{ }^{\mathbf{2 4 2}}$ isotope (41.999\%), then $\mathrm{Pu}^{239}$ isotope (37.441\%), $\mathrm{Pu}^{\mathbf{2 4 1}}$ isotope (15.218\%) and the last $\mathrm{Am}^{\mathbf{2 4 1}}$

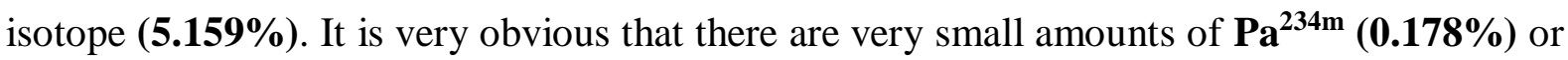


$\mathbf{U}^{235}(\mathbf{0 . 0 0 4 \%})$ in the samples. This clearly indicates that the samples are mainly Pu samples for research applications and most probably for alpha spectrometry [6].

As a part of the samples characterization, the masses of the radioisotopes existed in the samples were determined. The mass of an isotope in ( $\mathrm{gm})$ is given as:

$$
\begin{gathered}
\boldsymbol{m}_{\text {mass of isotope }}=\frac{N}{N_{A}} \times \text { Mass number of isotope } \\
\therefore m_{\text {mass of isotope }}=\frac{1}{\text { mole }^{-1}} \times \mathrm{gm} \cdot \text { mole }^{-1}=g m
\end{gathered}
$$

Substituting the basic decay equation:

$$
A=N \lambda
$$

where:

$\boldsymbol{A}$ is the activity of the isotope, $\boldsymbol{N}$ is the number of atoms and $\boldsymbol{\lambda}$ is the decay constant of the isotope $\left(s^{-1}\right)$.

$$
\therefore \boldsymbol{m}_{\text {mass of isotope }}=\frac{A}{N_{A} \cdot \lambda} \times \text { Mass number of the isotope }
$$

where $\boldsymbol{N}_{\boldsymbol{A}}$ is Avogadro's number $\left(\boldsymbol{N}_{\boldsymbol{A}}=\mathbf{6 . 0 2 2} \times \mathbf{1 0}^{\mathbf{2 3}} \mathbf{\text { mole }} \mathbf{e}^{\mathbf{1}}\right)$ and the mass number of intersted isotopes and it's decay constant have been illustrated in table 3.

Table 3: The mass number of the interested isotopes and their decay constant.

\begin{tabular}{|c|c|c|}
\hline Isotope & Mass number of the isotope & Decay constant \\
\hline $\mathrm{Pu}^{239}$ & 239 & $9.11 \mathrm{E}-13$ \\
\hline $\mathrm{Am}^{241}$ & 241 & $5.08 \mathrm{E}-11$ \\
\hline $\mathrm{Pu}^{241}$ & 241 & $1.53 \mathrm{E}-9$ \\
\hline $\mathrm{Pu}^{242}$ & 242 & $5.88 \mathrm{E}-14$ \\
\hline $\mathrm{U}^{235}$ & 235 & $3.12 \mathrm{E}-17$ \\
\hline $\mathrm{Pa}^{234 \mathrm{~m}}$ & 234 & $9.87 \mathrm{E}-3$ \\
\hline
\end{tabular}

The masses of intersted isotopes (gm) in the understudy samples have been calculated by substitution into eq.5 with different parameters. Table 4 has illustrated the masses of intersted isotopes (gm) in the understudy samples. According to table $\mathbf{4}$, the average mass ratios of each isotope in the samples were calculated and represented in figure 3. 
Table 4: The mass of the measured radioisotopes (mass \pm error\%) in gm.

\begin{tabular}{|c|c|c|c|c|c|c|}
\hline $\begin{array}{c}\text { Sample } \\
\text { codes }\end{array}$ & $\mathbf{P u}^{241}$ & $\mathrm{Am}^{241}$ & $\mathbf{P u}^{239}$ & $\mathbf{P u}^{242}$ & $\mathbf{U}^{235}$ & $\mathbf{P a}^{234 \mathrm{~m}}$ \\
\hline N6140 & $\begin{array}{c}5.854 \mathrm{E}-10 \\
\pm 2.106\end{array}$ & $\begin{array}{l}3.926 \mathrm{E}-09 \\
\pm 1.810 \mathrm{E}-3\end{array}$ & $\begin{array}{l}1.685 \mathrm{E}-06 \\
\pm 1.918 \mathrm{E}-2\end{array}$ & $\begin{array}{c}4.762 \mathrm{E}-05 \\
\pm 12.453 \mathrm{E}-2\end{array}$ & $\begin{array}{c}6.478 \mathrm{E}-06 \\
\pm 1.724\end{array}$ & $\begin{array}{l}1.251 \mathrm{E}-18 \\
\pm 6.900 \mathrm{E}-3\end{array}$ \\
\hline N6141 & $\begin{array}{c}3.068 \mathrm{E}-10 \\
\pm 8.149\end{array}$ & $\begin{array}{l}3.644 \mathrm{E}-09 \\
\pm 2.000 \mathrm{E}-3\end{array}$ & $\begin{array}{l}1.547 \mathrm{E}-06 \\
\pm 2.145 \mathrm{E}-2\end{array}$ & $\begin{array}{c}1.788 \mathrm{E}-05 \\
\pm 31.393 \mathrm{E}-2\end{array}$ & $\begin{array}{c}7.385 \mathrm{E}-06 \\
\pm 1.452\end{array}$ & $\begin{array}{r}6.657 \mathrm{E}-19 \\
\pm 13.300 \mathrm{E}-3\end{array}$ \\
\hline N6142 & $\begin{array}{c}4.554 \mathrm{E}-10 \\
\pm 9.919\end{array}$ & $\begin{array}{l}3.841 \mathrm{E}-09 \\
\pm 1.810 \mathrm{E}-3\end{array}$ & $\begin{array}{l}1.695 \mathrm{E}-06 \\
\pm 1.859 \mathrm{E}-2\end{array}$ & $\begin{array}{l}6.281 \mathrm{E}-05 \\
\pm 6.467 \mathrm{E}-2\end{array}$ & $\begin{array}{c}7.959 \mathrm{E}-06 \\
\pm 1.368\end{array}$ & $\begin{array}{l}1.149 \mathrm{E}-18 \\
\pm 7.320 \mathrm{E}-3\end{array}$ \\
\hline N6144 & $\begin{array}{c}2.313 \mathrm{E}-10 \\
\pm 38.405\end{array}$ & $\begin{array}{l}3.592 \mathrm{E}-09 \\
\pm 1.990 \mathrm{E}-3\end{array}$ & $\begin{array}{l}1.592 \mathrm{E}-06 \\
\pm 2.036 \mathrm{E}-2\end{array}$ & $\begin{array}{c}4.070 \mathrm{E}-05 \\
\pm 13.781 \mathrm{E}-2\end{array}$ & $\begin{array}{c}7.263 \mathrm{E}-06 \\
\pm 1.541\end{array}$ & $\begin{array}{l}8.802 \mathrm{E}-19 \\
\pm 9.830 \mathrm{E}-3\end{array}$ \\
\hline N6146 & $\begin{array}{c}8.015 \mathrm{E}-10 \\
\pm 3.150\end{array}$ & $\begin{array}{l}5.513 \mathrm{E}-09 \\
\pm 1.320 \mathrm{E}-3\end{array}$ & $\begin{array}{l}2.042 \mathrm{E}-06 \\
\pm 1.617 \mathrm{E}-2\end{array}$ & $\begin{array}{l}7.994 \mathrm{E}-05 \\
\pm 6.476 \mathrm{E}-2\end{array}$ & $\begin{array}{c}7.109 \mathrm{E}-06 \\
\pm 1.606\end{array}$ & $\begin{array}{c}5.391 \mathrm{E}-19 \\
\pm 16.360 \mathrm{E}-3\end{array}$ \\
\hline N6147 & $\begin{array}{c}3.514 \mathrm{E}-10 \\
\pm 18.455\end{array}$ & $\begin{array}{l}5.680 \mathrm{E}-09 \\
\pm 1.230 \mathrm{E}-3\end{array}$ & $\begin{array}{l}2.309 \mathrm{E}-06 \\
\pm 1.372 \mathrm{E}-2\end{array}$ & $\begin{array}{l}4.826 \mathrm{E}-05 \\
\pm 8.465 \mathrm{E}-2\end{array}$ & $\begin{array}{c}6.717 \mathrm{E}-06 \\
\pm 1.633\end{array}$ & $\begin{array}{l}8.691 \mathrm{E}-19 \\
\pm 9.750 \mathrm{E}-3\end{array}$ \\
\hline N6148 & $\begin{array}{c}4.155 \mathrm{E}-10 \\
\pm 4.075\end{array}$ & $\begin{array}{l}5.224 \mathrm{E}-09 \\
\pm 1.350 \mathrm{E}-3\end{array}$ & $\begin{array}{l}2.388 \mathrm{E}-06 \\
\pm 1.340 \mathrm{E}-2\end{array}$ & $\begin{array}{c}3.102 \mathrm{E}-05 \\
\pm 19.468 \mathrm{E}-2\end{array}$ & $\begin{array}{c}7.517 \mathrm{E}-06 \\
\pm 1.475\end{array}$ & $\begin{array}{l}1.109 \mathrm{E}-18 \\
\pm 7.720 \mathrm{E}-3\end{array}$ \\
\hline N6149 & $\begin{array}{c}4.331 \mathrm{E}-10 \\
\pm 7.507\end{array}$ & $\begin{array}{c}6.003 \mathrm{E}-09 \\
\pm 1.1150 \mathrm{E}-3\end{array}$ & $\begin{array}{l}2.757 \mathrm{E}-06 \\
\pm 1.113 \mathrm{E}-2\end{array}$ & $\begin{array}{l}4.750 \mathrm{E}-05 \\
\pm 7.878 \mathrm{E}-2\end{array}$ & $\begin{array}{c}7.521 \mathrm{E}-06 \\
\pm 1.440\end{array}$ & $\begin{array}{l}1.258 \mathrm{E}-18 \\
\pm 6.650 \mathrm{E}-3\end{array}$ \\
\hline N6150 & $\begin{array}{c}7.243 \mathrm{E}-10 \\
\pm 4.127\end{array}$ & $\begin{array}{l}5.146 \mathrm{E}-09 \\
\pm 1.350 \mathrm{E}-3\end{array}$ & $\begin{array}{l}2.091 \mathrm{E}-06 \\
\pm 1.510 \mathrm{E}-2\end{array}$ & $\begin{array}{l}4.496 \mathrm{E}-05 \\
\pm 7.547 \mathrm{E}-2\end{array}$ & $\begin{array}{c}7.343 \mathrm{E}-06 \\
\pm 1.487\end{array}$ & $\begin{array}{l}1.442 \mathrm{E}-18 \\
\pm 5.850 \mathrm{E}-3\end{array}$ \\
\hline N6151 & $\begin{array}{c}3.004 \mathrm{E}-10 \\
\pm 10.982\end{array}$ & $\begin{array}{l}4.901 \mathrm{E}-09 \\
\pm 1.510 \mathrm{E}-3\end{array}$ & $\begin{array}{l}2.276 \mathrm{E}-06 \\
\pm 1.474 \mathrm{E}-2\end{array}$ & $\begin{array}{c}2.715 \mathrm{E}-05 \\
\pm 21.403 \mathrm{E}-2\end{array}$ & $\begin{array}{c}7.468 \mathrm{E}-06 \\
\pm 1.555\end{array}$ & $\begin{array}{l}1.024 \mathrm{E}-18 \\
\pm 8.760 \mathrm{E}-3\end{array}$ \\
\hline N6152 & $\begin{array}{c}3.701 \mathrm{E}-10 \\
\pm 12.813\end{array}$ & $\begin{array}{l}5.637 \mathrm{E}-09 \\
\pm 1.260 \mathrm{E}-3\end{array}$ & $\begin{array}{l}2.261 \mathrm{E}-06 \\
\pm 1.420 \mathrm{E}-2\end{array}$ & $\begin{array}{l}4.554 \mathrm{E}-05 \\
\pm 8.373 \mathrm{E}-2\end{array}$ & $\begin{array}{c}8.231 \mathrm{E}-06 \\
\pm 1.350\end{array}$ & $\begin{array}{l}1.406 \mathrm{E}-18 \\
\pm 6.110 \mathrm{E}-3\end{array}$ \\
\hline N6153 & $\begin{array}{c}1.048 \mathrm{E}-09 \\
\pm 2.596\end{array}$ & $\begin{array}{l}1.166 \mathrm{E}-08 \\
\pm 6.199 \mathrm{E}-4\end{array}$ & $\begin{array}{l}4.462 \mathrm{E}-06 \\
\pm 7.340 \mathrm{E}-3\end{array}$ & $\begin{array}{c}3.364 \mathrm{E}-05 \\
\pm 15.178 \mathrm{E}-2\end{array}$ & $\begin{array}{c}7.236 \mathrm{E}-06 \\
\pm 1.567\end{array}$ & $\begin{array}{l}1.398 \mathrm{E}-18 \\
\pm 6.270 \mathrm{E}-3\end{array}$ \\
\hline N6154 & $\begin{array}{c}6.468 \mathrm{E}-10 \\
\pm 4.426\end{array}$ & $\begin{array}{l}1.021 \mathrm{E}-08 \\
\pm 7.048 \mathrm{E}-4\end{array}$ & $\begin{array}{l}3.719 \mathrm{E}-06 \\
\pm 8.760 \mathrm{E}-3\end{array}$ & $\begin{array}{l}4.521 \mathrm{E}-05 \\
\pm 8.905 \mathrm{E}-2\end{array}$ & $\begin{array}{c}6.912 \mathrm{E}-06 \\
\pm 1.633\end{array}$ & $\begin{array}{c}8.356 \mathrm{E}-19 \\
\pm 10.440 \mathrm{E}-3\end{array}$ \\
\hline N6155 & $\begin{array}{c}8.619 \mathrm{E}-10 \\
\pm 1.927\end{array}$ & $\begin{array}{l}9.975 \mathrm{E}-09 \\
\pm 7.131 \mathrm{E}-4\end{array}$ & $\begin{array}{l}3.501 \mathrm{E}-06 \\
\pm 9.200 \mathrm{E}-3\end{array}$ & $\begin{array}{c}2.219 \mathrm{E}-05 \\
\pm 24.637 \mathrm{E}-2\end{array}$ & $\begin{array}{c}8.599 \mathrm{E}-06 \\
\pm 1.298\end{array}$ & $\begin{array}{l}9.979 \mathrm{E}-19 \\
\pm 8.640 \mathrm{E}-3\end{array}$ \\
\hline
\end{tabular}




\begin{tabular}{|c|c|c|c|c|c|c|}
\hline N6156 & $\begin{array}{c}1.499 \mathrm{E}-09 \\
\pm 1.021\end{array}$ & $\begin{array}{l}1.034 \mathrm{E}-08 \\
\pm 6.958 \mathrm{E}-4\end{array}$ & $\begin{array}{l}3.912 \mathrm{E}-06 \\
\pm 8.330 \mathrm{E}-3\end{array}$ & $\begin{array}{l}7.807 \mathrm{E}-05 \\
\pm 4.245 \mathrm{E}-2\end{array}$ & $\begin{array}{c}7.789 \mathrm{E}-06 \\
\pm 1.449\end{array}$ & $\begin{array}{l}1.585 \mathrm{E}-18 \\
\pm 5.500 \mathrm{E}-3\end{array}$ \\
\hline & $\begin{array}{c}2.313 \mathrm{E}-10 \\
\pm 38.405\end{array}$ & $\begin{array}{r}3.592 \mathrm{E}-09 \\
\pm 1.990 \mathrm{E}-3\end{array}$ & $\begin{array}{r}1.547 \mathrm{E}-06 \\
\pm 2.145 \mathrm{E}-2\end{array}$ & $\begin{array}{c}1.788 \mathrm{E}-05 \\
\pm 31.393 \mathrm{E}-2\end{array}$ & $\begin{array}{c}6.478 \mathrm{E}-06 \\
\pm 1.724\end{array}$ & $\begin{array}{c}5.391 \mathrm{E}-19 \\
\pm 16.360 \mathrm{E}-3\end{array}$ \\
\hline Range & $\begin{array}{c}- \\
1.499 \mathrm{E}-09 \\
\pm 1.021\end{array}$ & $\begin{array}{c}- \\
1.166 \mathrm{E}-08 \\
\pm 6.199 \mathrm{E}-4\end{array}$ & $\begin{array}{c}- \\
4.462 \mathrm{E}-06 \\
\pm 7.340 \mathrm{E}-3\end{array}$ & $\begin{array}{c}- \\
7.994 \mathrm{E}-05 \\
\pm 6.476 \mathrm{E}-2\end{array}$ & $\begin{array}{c}- \\
8.599 \mathrm{E}-06 \\
\pm 1.298\end{array}$ & $\begin{array}{c}- \\
1.585 \mathrm{E}-18 \\
\pm 5.500 \mathrm{E}-3\end{array}$ \\
\hline Average & $\begin{array}{c}6.02 \mathrm{E}-10 \\
\pm 8.644\end{array}$ & $\begin{array}{r}6.35 \mathrm{E}-09 \\
+1.301 \mathrm{E}-3\end{array}$ & $\begin{array}{r}2.55 \mathrm{E}-06 \\
\pm 1.413 \mathrm{E}-2\end{array}$ & $\begin{array}{r}4.48 \mathrm{E}-05 \\
+13.111 \mathrm{E}-2\end{array}$ & $\begin{array}{c}7.44 \mathrm{E}-06 \\
\pm 1.505\end{array}$ & $\begin{array}{r}1.09 \mathrm{E}-18 \\
\pm 8.628 \mathrm{E}-3\end{array}$ \\
\hline
\end{tabular}

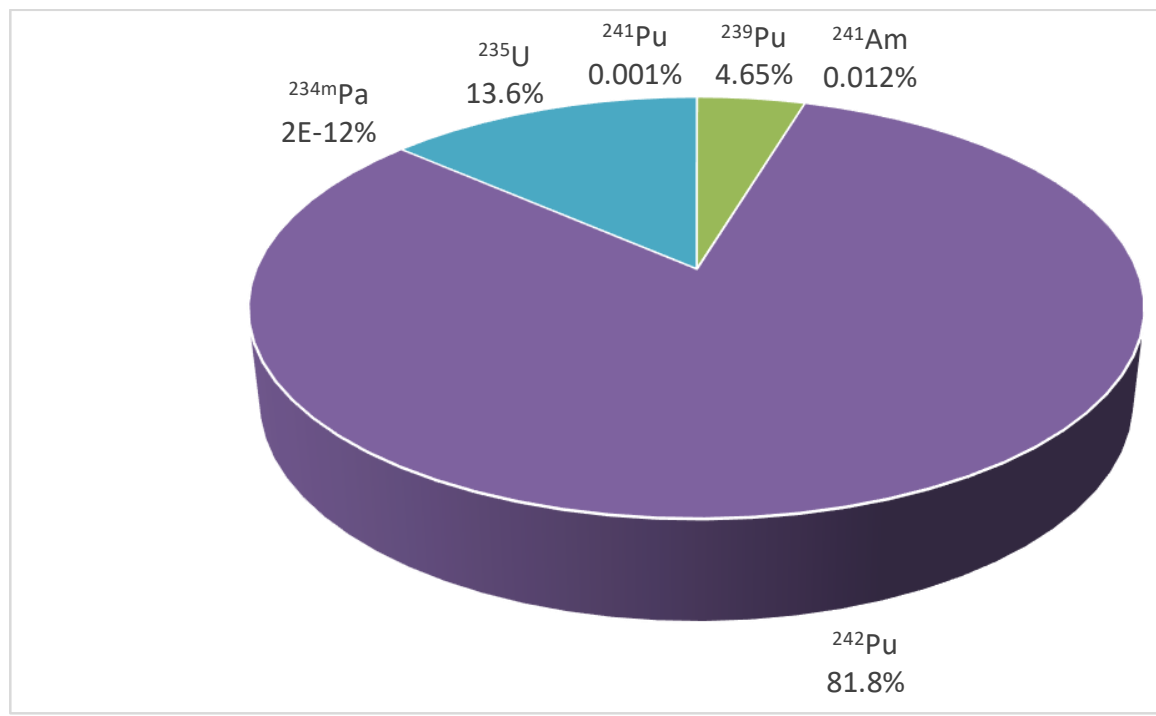

Figure 3: The average mass ratios of the measured isotopes found in the samples

From figure 3, it can be seen that the masses in the samples are in the following order: ${ }^{242} \mathrm{Pu}$ isotope $(\mathbf{8 1 . 8 \%}),{ }^{235} \mathrm{U}$ isotope $(\mathbf{1 3 . 6 \%}),{ }^{239} \mathrm{Pu}$ isotope (4.65\%), ${ }^{241} \mathrm{Am}$ isotope $(\mathbf{0 . 0 1 2 \%})$ and the ${ }^{241} \mathbf{P u}$ isotope $\mathbf{( 0 . 0 0 1 \%}$ ). It is very obvious that there are very small amounts of ${ }^{{ }^{234 m}} \mathbf{P a}$ isotope in the samples (2E-12\%). According to the masses of the isotopes we can categorize the nuclear material for the nuclear seecurity and nuclear forensics applications. It is obvious that the masses calculated are low and lower than $15 \mathrm{gm}$ which make them below category 3 $(<\mathrm{Cat} 3)[7]$.

It can be also noticed that this very small quantity of $\mathrm{Pu}$ is difficult to be measured via chemical analysis. Additionally, analytical chemical determination of the nuclear material $(\mathrm{NM})$ content requires disolution of the samples, leading to sample destruction. This is an 
advantage of non-distructive analysis (NDA) of extracting information while avoiding such destruction.

\section{Conclusion:}

The use of gamma spectrometry in nuclear forensic applications is often motivated by the desire to either cheaply, quickly and non-destructively categorize nuclear and other radioactive materials in terms of their isotopic composition or to characterize them in terms of their elemental composition. The experimental results of non-destructive gamma analysis and ISOCS system Falcon 5000 have been used to characterize plutonium samples. The proposed measuring method is effective for characterizing the nuclear material especially in case of samples with of very small quantity.

\section{References:}

[1] IAEA, "Application of nuclear forensics in combating illicit trafficking of nuclear and other radioactive material”, IAEA-t Ecdoc series, (2013), Issn 1011-4289, no. 1730.

[2] M. Wallenius and K. Mayer "Age determination of plutonium material in nuclear forensics by thermal ionization mass spectrometry", Fresenius J Anal Chem, 366: (2000) 234-238.

[3] J. A. C. Alvarado, F. Chawla and P. Froidevaux, "Determining ${ }^{241} \mathrm{Pu}$ in environmental samples: case studies in alpine Soils", (2011), Radio chim. Acta, 99:121-129.

[4] I. Lantzos, C. Kouvalaki and G. Nicolaou, "Plutonium fingerprinting in nuclear forensics of spent nuclear fuel", (2015), Progress in Nuclear Energy, 85:333-336.

[5] W. El-Gammal, H. Abu-Zied, A. Nada, Z. F. Akl, F. Ragab and M. Tawfeek, "Age determination of Pu-bearing samples using gamma spectrometry for safeguards and nuclear forensics applications", (2020), IOSR Journal of Applied Physics (IOSR-JAP), 12:8-13.

[6] "A procedure for the rapid determination of Pu isotopes and Am-241 in soil and sediment samples by alpha spectrometry", Analytical Quality in Nuclear Applications No. IAEA/AQ/11, IAEA, Vienna, (2009), ISSN 2074-7659.

[7] "NSS.13. Nuclear security recommendations on physical protection of nuclear material and nuclear facilities (INFCIRC/225/revision 5)", IAEA nuclear security series, (2010), ISSN 1816-9317. 


\section{الملخص العربي للبحث}

عنوان البحث "توصيف مواد منطوية على بلوتونيوم باستخدام قياسات لا إتلافية متعلقة بالأدلة الجنائية النووية" مروة توفيق(*) - و وائل الجمال 1 - حننية أبو زيد² - عفاف ندا2 - زينب حسن 1 ـ فاطمة رجب2

$$
\begin{aligned}
& 1 \text { هيئة الرقابة النووية والإشعاعية (ENRRA) - القاهرة - مصر. } \\
& 2 \text { كلية البنات للآداب والعلوم والتربية - جامعة عين شمس - القاهرة - مصر. }
\end{aligned}
$$

أحد المكونات الرئيسية التي يجب وضعها للتحكم في المو اد النووية وتطبيقاتها هو استخدام نظام قياس قوي. وظيفة هذا النظام ليس فقط التحقق من صحة المعلومات المتعلقة بالمو اد النووية ولكن يجب أن يكون قادراً على تمييز المواد الغير معروفة أيضاً. الخصائص الكيميائية و الفيزيائية المتخلصة من عملية التوصيف ضرورية لتحديد الاستخدام المقصود ومنشأ

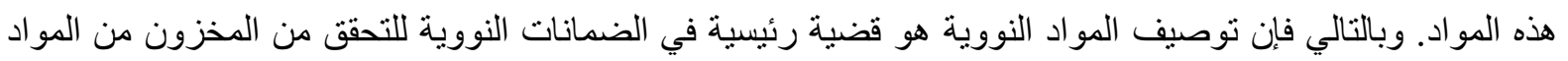

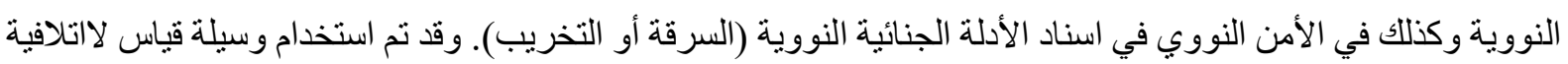

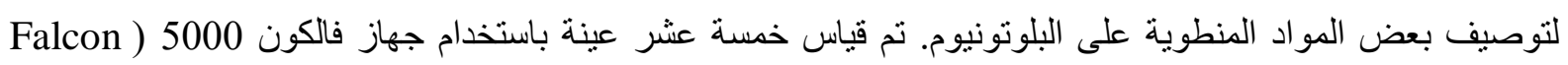

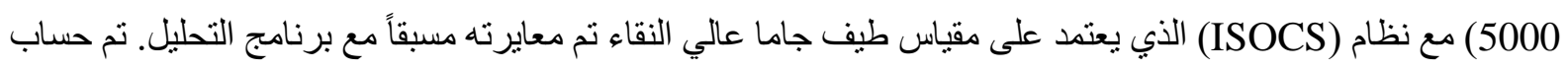

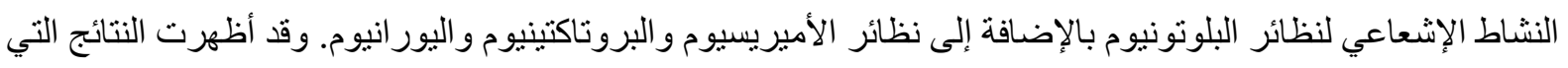
تم الحصول عليها أن طريقة القياس المقترحة يمكن استخدامها بنجاح لتوصيف عينات تنطوي على البلوتونيوم لأغر اض ولاض الأدلة الجنائية النووية. 